A16

doi: 10.14232/ syrpharmacognosy.2021.a16

\title{
Ensifolins A-M, new phenanthrenes from Juncus ensifolius
}

\section{Dóra Stefkó}

Email: stefko.dori@gmail.com

Phenanthrenes form an increasing group of aromatic plant secondary metabolites. These compounds are derived mainly from stilbene precursors and can be divided into three major groups: mono-, di-, and triphenanthrenes. In many cases, phenanthrenes are substituted with special functional groups, therefore, they are characteristic to certain plant families; e.g. stilbene-substituted phenanthrenes were isolated only from Orchidaceae species, while vinylsubstituted phenanthrenes were found exclusively in Juncaceae species. These compounds can serve as chemotaxonomic markers. Phenanthrenes are representative metabolites of Juncaceae species [1].

In continuation of our work dealing with the isolation of phenanthrenes from Juncaceae species, Juncus ensifolius Wikstr. was investigated. 17 Phenanthrenes were isolated from the methanol extract of the plant by using different chromatographic methods (VLC, MPLC, Sephadex LH-20 gel chromatography and HPLC). The structure elucidation of the compounds was carried out by extensive NMR and HRMS experiments.

14 Compounds (ensifolins A-M), from which 8 monomers and 4 dimers are new natural products. Some compounds are unique as they are substituted with flavonoid or benzaldehyde. All compounds were isolated for the first time from the plant.

Supervisor: Andrea Vasas

\section{Acknowledgements:}

The work was supported by the UNKP-20-3 New National Excellence Program of the Ministry of Human capacities and EFOP 3.6.3-VEKOP-16-2017-00009.

\section{References}

[1] Tóth B, et al. J. Nat. Prod. 2018; 81: 661-678. 